\section{Esperança de vida ao nascer: impacto das variações na mortalidade por idade $e$ causas de morte no Município de Campinas, São Paulo, Brasil}

\author{
Life expectancy at birth: impact of variation in \\ mortality by age group and cause of death in \\ Campinas, São Paulo State, Brazil
}

1. Faculdade de Ciências
Médicas, Universidade
Estadual de Campinas,
Campinas, Brasil.
Correspondência
A. P. Belon
Faculdade de Ciências
Médicas, Universidade
Estadual de Campinas.
Rua Buarque de Macedo 101,
bloco3,apto.093, Campinas, SP
13073-010, Brasil.
paulabelon@gmail.com

Abstract

This study investigated the impact of variation in mortality by age group and cause of death on gains in life expectancy at birth in the city of Campinas, São Paulo State, Brazil, in 1991, 2000, and 2005. Life tables were constructed. Pollard's method was used to estimate the contributions by age group and cause of death on gains in life expectancy. In 1991-2000, the age group that most contributed was $0-1$ year $(31.1 \%$ for males and 22.9\% for females). In 2000-2005, 79\% of the gain for males was the result of mortality improvements in the 15-44-year bracket. Cardiovascular diseases made the largest contribution in 1991-2000 (66.1\% for males and 43.5\% for females). A loss in longevity was seen in men (1.1 year) resulting from increased mortality from external causes. In 2000-2005, the substantial gain (2.3 year) in male life expectancy was due to a reduction in mortality from external causes. Neoplasms had a negative effect on the gain (0.11 year for males and 0.15 for females). These findings should help support public health policies to reduce mortality risks and increase life expectancy.

Life Expectancy at Birth; Mortality; Cause of Death; Age Groups
Ana Paula Belon 1

Marilisa Berti de Azevedo Barros 1

\section{Introdução}

A esperança de vida ao nascer é amplamente utilizada como medida do nível de mortalidade de uma população e como indicador sintético da qualidade de saúde e de vida de países e regiões. Por não ser influenciada pelos efeitos da estrutura etária, a esperança de vida ao nascer é uma ferramenta útil no monitoramento da tendência da mortalidade e para examinar os diferenciais entre regiões e subgrupos sociodemográficos 1,2,3. Outra vantagem da esperança de vida ao nascer é a sua facilidade de compreensão, representando o número médio de anos que se espera que um recém-nascido viva, se mantidas as condições de mortalidade existentes na população residente no período de tempo considerado.

Estimativas apontam que, a partir do século $\mathrm{XX}$, houve expressivo crescimento da esperança de vida ao nascer e diminuição das desigualdades do tempo médio de vida entre os países 4,5 . Por volta de 1800, o tempo médio de vida não ultrapassava os 30 anos de idade e no final do século XX atingia o patamar de 67 anos ${ }^{6}$. No Brasil, a esperança de vida ao nascer que era, em 1940, de apenas 44,9 anos passa para 68,6 anos no ano de 2000 2. Como destaca a literatura científica, diversos fatores, como renda, educação, cobertura dos serviços de saúde e de saneamento básico, entre outros, influenciaram a queda da mortalidade, promovendo o aumento da esperança de vida ao nascer $1,2,4,5$. 
Apesar de ser extensamente conhecida a relação entre a mortalidade e a expectativa de vida ao nascer, a natureza desta é complexa ${ }^{7}$. As transformações nos padrões saúde-doença resultam em mudanças na estrutura da mortalidade por idade, de forma que a queda dos coeficientes de mortalidade não ocorre uniformemente entre as idades, devido às diferentes taxas de incidência de causas de morte segundo grupo etário e sexo 3 . Desse modo, para mensurar e explicar a mudança na esperança de vida ao nascer, é necessário examinar o comportamento da variação da mortalidade nas idades e nas causas de morte e no consequente aumento da esperança de vida ao nascer 7 .

Esta análise pode ser realizada através do método proposto por Pollard 7. Interessado na relação exata entre mortalidade e esperança de vida, Pollard desenvolveu um conjunto de equações que permitem analisar os efeitos das variações da mortalidade sobre a esperança de vida, ao identificar as contribuições de cada faixa etária e de cada grupo de causas de morte no aumento da vida média em um intervalo de tempo em uma dada população. A estimativa dessas contribuições permite subsidiar o planejamento e avaliação de políticas públicas de saúde, orientando esforços em ações mais específicas na prevenção e controle de determinadas doenças e agravos à saúde.

Portanto, o objetivo deste estudo é avaliar o impacto da tendência dos coeficientes de mortalidade por grupos etários e por causas de morte segundo sexo na variação da esperança de vida ao nascer de residentes do Município de Campinas, Estado de São Paulo, Brasil, nos anos de 1991, 2000 e 2005.

\section{Métodos}

Trata-se de um estudo ecológico descritivo que emprega dados secundários referentes ao $\mathrm{Mu}$ nicípio de Campinas dos anos de 1990 a 1992, 1999 a 2001 e 2004 a 2006, para a construção de tábuas de mortalidade e a aplicação do método de Pollard 7. A escolha do recorte temporal para avaliar a tendência se deve ao fato de 1991 e 2000 serem anos censitários e de 2005 ser o ano central da década. Os dados de óbitos e nascidos vivos foram obtidos do Sistema de Informações sobre Mortalidade (SIM) e do Sistema de Informações sobre Nascidos Vivos (SINASC), respectivamente. Para o período de 1990 a 1992 e 1999 a 2001, foram utilizados os registros de estatísticas vitais disponibilizados pelo Departamento de Informática do SUS (DATASUS; http://www.datasus.gov. br) do Ministério da Saúde, e os dados de 2004 a
2006 são provenientes da Secretaria Municipal de Saúde de Campinas. Dos censos demográficos de 1991 e 2000 do Instituto Brasileiro de Geografia e Estatística (IBGE; http://www.ibge.gov.br), foram extraídos os dados populacionais. Para estes anos, foram estimados os tamanhos da população para 1o de julho. As estimativas populacionais para o meio do ano de 2005 foram calculadas pelo método $\mathrm{AiBi}$, que permite produzir projeções para pequenas áreas com base na tendência do crescimento populacional da área maior em que estas se inserem ${ }^{8}$.

Foram calculados os coeficientes específicos de mortalidade por idade, sendo que, para os maiores de 1 ano de idade, foi utilizada no numerador a média trienal dos óbitos por grupo etário de cada ano central analisado (1991, 2000 e 2005). O coeficiente de mortalidade infantil foi obtido da relação entre a soma de três anos consecutivos de óbitos de menores de 1 ano e a de nascidos vivos. Foram utilizados grupos etários quinquenais, com exceção dos iniciais (0-1 ano e de 1-4 anos de idade) e do último (80 anos ou mais).

A categorização das causas básicas de morte obedeceu a duas versões da Classificação Internacional das Doenças (CID), vigentes no período estudado: 9a (CID-9) e 10a revisão (CID-10). Foram selecionadas as seis causas básicas de morte com maior expressão, em termos proporcionais, nos três anos investigados. Os grupos de causas de morte analisados foram: doenças infecto-parasitárias (capítulo 1 da CID-9 e da CID-10), neoplasias (capítulo 2 da CID-9 e CID-10), doenças do aparelho circulatório (capítulo 7 da CID-9 e capítulo 9 da CID-10), doenças do aparelho respiratório (capítulo 8 da CID-9 e capítulo 10 da CID-10), causas mal-definidas (capítulo 16 da CID-9 e capítulo 18 da CID-10) e causas externas (capítulo 17 da CID-9 e capítulo 20 da CID-10).

Tábuas de mortalidade foram construídas para homens, mulheres e ambos os sexos para 1991, 2000 e 2005. Foi utilizado o modelo abreviado da tábua de mortalidade, no qual foram empregados intervalos etários quinquenais (salvo os grupos etários de 0-1 ano e 1-4 anos) e adotado 80 anos ou mais como agrupamento final. Em sua construção, foram convertidos os coeficientes específicos de mortalidade entre as idades $\mathrm{x} e$ $\mathrm{x}+\mathrm{n}$ na função de probabilidade de morte entre as duas idades exatas, ${ }_{n} q_{x}$. A partir desta, foram derivadas as demais funções da tábua de mortalidade, tais como o número de sobreviventes à idade $\mathrm{x}\left(l_{x}\right)$ e o número de óbitos entre as idades $\mathrm{x}$ e $\mathrm{x}+\mathrm{n}\left({ }_{n} d_{x}\right)$. Para compor o total de anos vividos $\left({ }_{n} L_{x}\right)$ pelo grupo etário de 0 - 1 ano de idade e de 1-4 anos, o tempo vivido por cada pessoa foi estimado a partir do fator de separação de óbi- 
tos. Foram calculados fatores de separação para a população total e para a masculina e feminina segundo o método proposto por Arriaga 9 que considera a data de nascimento e de óbito e a fração do tempo vivido e do tempo de morte.

A partir da tábua de mortalidade, obteve-se a expectativa de vida ao nascer e em cada idade exata, as quais foram utilizadas no método de decomposição de Pollard. O método foi aplicado para a população total, a masculina e a feminina para os períodos 1991 a 2000 e 2000 a 2005 . As análises dos efeitos da mortalidade sobre o aumento da expectativa de vida foram realizadas para os agrupamentos etários e de causas de morte definidos anteriormente.

O método de decomposição de Pollard 7 é constituído por um conjunto de relações matemáticas que permitem desagregar a diferença da expectativa de vida ao nascer entre dois períodos em contribuições dos níveis de mortalidade por grupos etários e por causas de morte. Ou seja, o método mensura o impacto das mudanças dos níveis de mortalidade segundo grupos etários e causas de morte na variação da expectativa de vida ao nascer entre dois períodos.

A contribuição de grupos etários no aumento da expectativa de vida ao nascer entre o tempo 01 e o tempo 02 foi estimada pela seguinte equação:

$$
e_{0}^{01}-e_{0}^{02}=\sum\left({ }_{n} Q_{x}^{01}-{ }_{n} Q_{x}^{02}\right) * W_{x}
$$

Onde, $e_{0}^{01}=$ expectativa de vida ao nascer no tempo 01; $e_{0}^{02}=$ expectativa de vida ao nascer no tempo $02 ;{ }_{n} Q_{x}=$ força de mortalidade entre x e $\mathrm{x}+\mathrm{n} ; W_{x}=$ peso da idade $\mathrm{x}$.

A força de mortalidade entre x e $\mathrm{x}+\mathrm{n},{ }_{n} Q_{x}$, foi obtida a partir da seguinte fórmula:

$$
{ }_{n} Q_{x}=-\ln \left(l_{x+n} / l_{x}\right)
$$

O peso da idade, $W_{x}$, foi calculado por:

$$
W_{x}=0,5 *\left({ }_{x} p_{0}^{01} * e_{0}^{02}+{ }_{x} p_{0}^{02} * e_{0}^{01}\right)
$$

Sendo que a probabilidade de sobreviver do nascimento até a idade $\mathrm{x},{ }_{x} p_{0}$, foi dada por:

$$
{ }_{x} p_{0}=l_{x} / l_{0}
$$

Em relação à contribuição dos grupos de causas de morte, segundo grupos etários, no ganho da esperança de vida ao nascer, o método parte do pressuposto de que há independência da mortalidade por causas de morte. Esta contribuição foi calculada a partir da relação:

$$
\begin{aligned}
& e_{0}^{01}-e_{0}^{02}=\sum\left({ }_{1} Q_{0}^{(i) 01}-{ }_{1} Q_{0}^{(i) 02}\right) * W_{0}+ \\
& \sum\left({ }_{4} Q_{1}^{(i) 01}-{ }_{4} Q_{0}^{(i) 02}\right) * W_{2}+ \\
& \sum\left({ }_{5} Q_{5}^{(i) 01}-{ }_{5} Q_{5}^{(i) 02}\right) * W_{7,5}+\ldots
\end{aligned}
$$

Onde, ${ }_{n} Q_{x}^{(i)}=$ probabilidade de morte pela causa i entre as idades $\mathrm{x}$ e $\mathrm{x}+\mathrm{n}$

Esta função foi estimada por:

$$
{ }_{n} Q_{x}^{(i)}={ }_{n} Q_{x} *\left({ }_{n} D_{x}^{(i)} /{ }_{n} D_{x}\right)
$$

Sendo, ${ }_{n} D_{x}^{(i)}=$ número de óbitos pela causa $\mathrm{i}$ entre $\mathrm{x}$ e $\mathrm{x}+\mathrm{n}$.

Através deste método 7, foram calculadas as contribuições positivas (ganhos) e negativas (perdas) na variação da esperança de vida ao nascer, as quais foram analisadas a partir de valores absolutos (expresso em anos) e de porcentagens.

\section{Resultados}

A esperança de vida ao nascer no Município de Campinas passou de 69,9 anos em 1991 para 74,7 anos em 2005, representando um aumento de 4,8 anos. No período estudado, o sexo feminino apresentou a maior expectativa de vida. Em 1991, as mulheres viveriam em média 74,3 anos. Em 2005, este indicador aumenta para 78,4 anos. Já entre os homens, embora o tempo médio de vida seja inferior ao das mulheres, o incremento foi ainda maior: 5,2 anos foram acrescidos entre 1991 e 2005. O ganho da esperança de vida ao nascer foi maior entre as mulheres no período de 1991 a 2000, e nos homens entre 2000 a 2005 (Tabela 1).

Estes aumentos da esperança de vida ao nascer são resultados da queda das probabilidades de morte $\left({ }_{n} q_{x}\right)$ no decorrer dos anos estudados. As curvas da Figura 1 indicam que a probabilidade de morte no primeiro ano de vida apresentou importante redução entre 1991 e 2005. Embora, entre a população de jovens, as probabilidades de morte tenham sofrido um aumento em 2000, foi registrada expressiva diminuição entre este ano e 2005. Também entre os idosos houve redução, ainda que menos significativa em comparação com outras faixas etárias.

A contribuição de cada grupo etário no aumento da esperança de vida ao nascer é apresentada na Tabela 2. Entre os homens, no período de 1991 a 2000, o maior responsável pelo incremento da expectativa de vida ao nascer foi o grupo dos menores de 1 ano de idade $(31,1 \%)$, e as idades acima de 45 anos responderam por cerca de $85 \%$ do crescimento total do indicador. Nas idades de 15-34 anos, o aumento dos coeficientes de mortalidade ocasionaram redução dos anos de vida a serem vividos. O grupo etário de 20-24 anos, por exemplo, foi responsável pela perda de 0,23 ano, gerando impacto negativo de $11 \%$ na variação do tempo médio de vida. Entre 2000-2005, este quadro se inverte. Foi a diminuição dos coefi- 


\section{Tabela 1}

Esperança de vida ao nascer, segundo o sexo. Campinas, São Paulo, Brasil, 1991, 2000 e 2005.

\begin{tabular}{lcccccc}
\hline \multirow{2}{*}{ Sexo } & \multicolumn{3}{c}{ Expectativa de vida ao nascer } & \multicolumn{3}{c}{ Anos incrementados } \\
& 1991 & $\mathbf{2 0 0 0}$ & $\mathbf{2 0 0 5}$ & $\mathbf{1 9 9 1 - 2 0 0 0}$ & $\mathbf{2 0 0 0 - 2 0 0 5}$ & $\mathbf{1 9 9 1 - 2 0 0 5}$ \\
& & & & & & \\
Total & 69,9 & 72,3 & 74,7 & 2,4 & 2,4 & 4,8 \\
Homens & 65,9 & 67,7 & 71,1 & 1,8 & 3,4 & 5,2 \\
Mulheres & 74,3 & 77,2 & 78,4 & 2,9 & 1,2 & 4,1 \\
\hline
\end{tabular}

Figura 1

Probabilidades de morte, por grupo etário. Campinas, São Paulo, Brasil, 1991, 2000 e 2005.

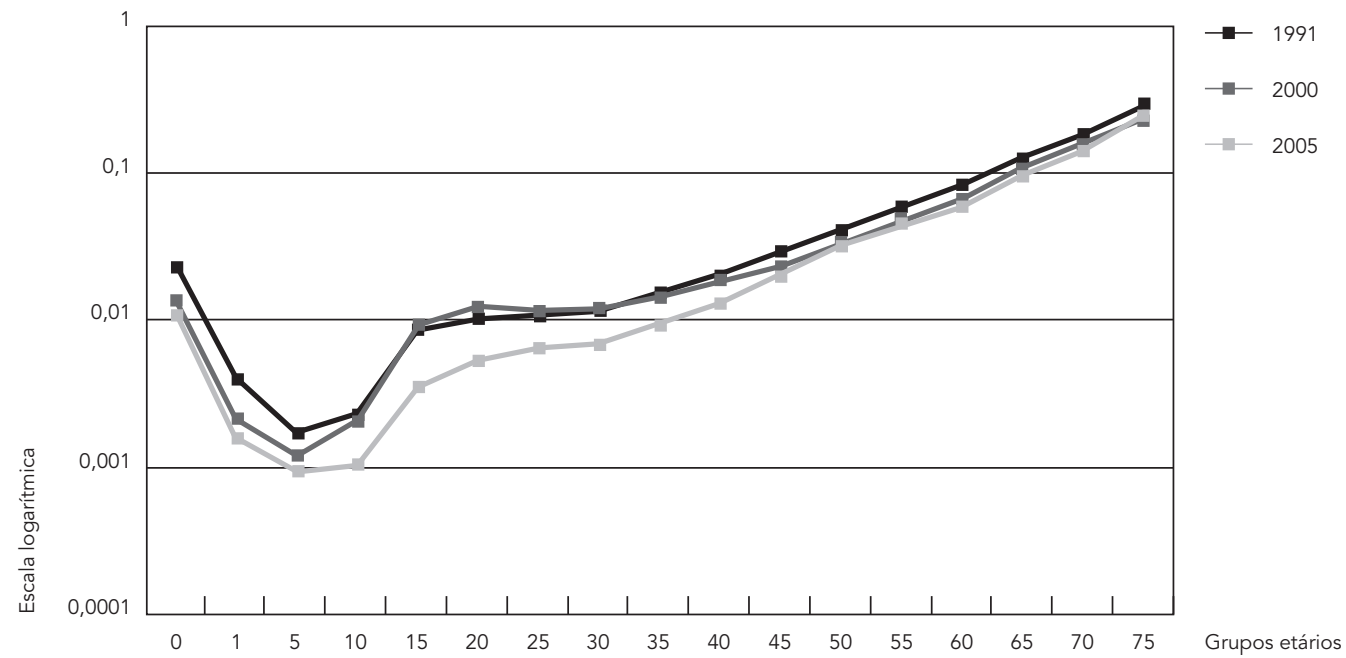

cientes de mortalidade nas idades de 15-44 anos que mais favoreceu o aumento da expectativa de vida ao nascer dos homens neste período, correspondendo a $79 \%$ de todo o crescimento registrado. No sexo feminino, reduções na mortalidade infantil e nos coeficientes das idades acima de 65 anos contribuíram expressivamente entre 1991 e 2000, perfazendo um total de $66,7 \%$ do ganho global da esperança de vida ao nascer. No período seguinte, os maiores ganhos concentraram-se nas faixas etárias de 0-1 ano, 25-39 anos e 55-74 anos. Em ambos os sexos, observa-se que, entre 1991 a 2000, somente os grupos etários de 15-34 contribuíram negativamente para a elevação da esperança de vida ao nascer. Entre 2000 e 2005, com a diminuição dos níveis de mortalidade em todas as idades, apenas os grupos etários acima de 75 anos reduziram o ganho total.

Na Tabela 3, encontra-se a contribuição de cada grupo de causas de morte no aumento da esperança de vida ao nascer. Entre 1991 e 2000, as causas externas foram responsáveis pela perda de um ano de vida nos homens. O declínio da mortalidade por neoplasias, doenças do aparelho circulatório e respiratório geraram as maiores contribuições no ganho da esperança de vida ao nascer dos homens e das mulheres nesse período. As neoplasias contribuíram com 0,28 ano no aumento da esperança de vida masculina e com 0,34 ano na feminina. A forte queda da mortalidade por doenças cardiovasculares, entre 1991 e 2000, resultou no acréscimo de aproximada- 
Contribuição em número de anos e em porcentagem dos grupos etários no aumento da esperança de vida ao nascer nos períodos de 1991 a 2000 e 2000 a 2005, segundo o sexo. Campinas, São Paulo, Brasil, 1991, 2000 e 2005.

\begin{tabular}{|c|c|c|c|c|c|c|}
\hline \multirow{3}{*}{$\begin{array}{l}\text { Grupo } \\
\text { etário } \\
\text { (anos) }\end{array}$} & \multicolumn{2}{|c|}{ Homens } & \multicolumn{2}{|c|}{ Mulheres } & \multicolumn{2}{|c|}{ Ambos os sexos } \\
\hline & $1991-2000$ & $2000-2005$ & $1991-2000$ & $2000-2005$ & $1991-2000$ & $2000-2005$ \\
\hline & Anos (\%) & Anos (\%) & Anos (\%) & Anos (\%) & Anos (\%) & Anos (\%) \\
\hline 0 & $0,6445(31,1)$ & $0,1648(4,9)$ & $0,7396(22,9)$ & $0,2347(26,4)$ & $0,6910(26,1)$ & $0,1995(9,0)$ \\
\hline 1 & $0,0975(4,7)$ & $0,0534(1,6)$ & $0,1678(5,2)$ & $0,0232(2,6)$ & $0,1302(4,9)$ & $0,0396(1,8)$ \\
\hline 5 & $0,0651(3,1)$ & $-0,0020(-0,1)$ & $-0,0014(0,0)$ & $0,0397(4,5)$ & $0,0348(1,3)$ & $0,0173(0,8)$ \\
\hline 10 & $0,0046(0,2)$ & $0,0816(2,4)$ & $0,0232(0,7)$ & $0,0605(6,8)$ & $0,0135(0,5)$ & $0,0720(3,3)$ \\
\hline 15 & $-0,1547(-7,5)$ & $0,5830(17,5)$ & $0,0755(2,3)$ & $0,0721(8,1)$ & $-0,0485(-1,8)$ & $0,3460(15,6)$ \\
\hline 20 & $-0,2276(-11,0)$ & $0,6511(19,5)$ & $0,0494(1,5)$ & $0,0512(5,8)$ & $-0,1022(-3,9)$ & $0,3755(16,9)$ \\
\hline 25 & $-0,1151(-5,6)$ & $0,3855(11,6)$ & $0,0435(1,3)$ & $0,0930(10,5)$ & $-0,0480(-1,8)$ & $0,2517(11,4)$ \\
\hline 30 & $-0,0705(-3,4)$ & $0,3541(10,6)$ & $0,0338(1,0)$ & $0,0821(9,2)$ & $-0,0266(-1,0)$ & $0,2293(10,4)$ \\
\hline 35 & $0,0697(3,4)$ & $0,2980(8,9)$ & $-0,0041(-0,1)$ & $0,0916(10,3)$ & $0,0402(1,5)$ & $0,2018(9,1)$ \\
\hline 40 & $-0,0009(0,0)$ & $0,3594(10,8)$ & $0,0890(2,8)$ & $0,0355(4,0)$ & $0,0488(1,8)$ & $0,2088(9,4)$ \\
\hline 45 & $0,1989(9,6)$ & $0,1466(4,4)$ & $0,1318(4,1)$ & $0,0560(6,3)$ & $0,1772(6,7)$ & $0,1053(4,8)$ \\
\hline 50 & $0,2060(10,0)$ & $0,0967(2,9)$ & $0,1503(4,7)$ & $-0,0331(-3,7)$ & $0,1902(7,2)$ & $0,0380(1,7)$ \\
\hline 55 & $0,3076(14,9)$ & $0,0219(0,7)$ & $0,1508(4,7)$ & $0,0836(9,4)$ & $0,2402(9,1)$ & $0,0503(2,3)$ \\
\hline 60 & $0,3566(17,2)$ & $0,1754(5,3)$ & $0,1642(5,1)$ & $0,0836(9,4)$ & $0,2736(10,3)$ & $0,1358(6,1)$ \\
\hline 65 & $0,1956(9,5)$ & $0,2008(6,0)$ & $0,3036(9,4)$ & $0,1126(12,7)$ & $0,2468(9,3)$ & $0,1625(7,3)$ \\
\hline 70 & $0,1584(7,7)$ & $0,1190(3,6)$ & $0,4005(12,4)$ & $0,1840(20,7)$ & $0,2754(10,4)$ & $0,1502(6,8)$ \\
\hline 75 & $0,2015(9,7)$ & $-0,1275(-3,8)$ & $0,4390(13,6)$ & $-0,1122(-12,6)$ & $0,3107(11,7)$ & $-0,1209(-5,5)$ \\
\hline 80 ou mais & $0,1323(6,4)$ & $-0,2309(-6,9)$ & $0,2720(8,4)$ & $-0,2706(-30,5)$ & $0,2013(7,6)$ & $-0,2471(-11,2)$ \\
\hline Total & $2,0695(100,0)$ & $3,3310(100,0)$ & $3,2283(100,0)$ & $0,8877(100,0)$ & $2,6488(100,0)$ & $2,2159(100,0)$ \\
\hline
\end{tabular}

mente 1,4 ano na esperança de vida ao nascer da população geral. As doenças do aparelho respiratório responderam por $25,6 \%$ e $11,6 \%$ do aumento do tempo médio de vida de homens e de mulheres, respectivamente.

No período de 2000 a 2005, verifica-se que o principal grupo de causas de morte que favoreceu o incremento da esperança de vida ao nascer masculina foi o das causas externas. Estas se tornaram responsáveis pela elevação de 2,3 anos no indicador, o que representa $69,7 \%$ do aumento total do tempo médio de vida dos homens. Para as mulheres, foram as mortes relacionadas às doenças do aparelho circulatório as que mais contribuíram com o aumento da esperança de vida ao nascer, sendo seguidas pelas causas externas.

As neoplasias provocaram uma redução, no ganho total do tempo médio de vida entre $2000 \mathrm{e}$ 2005, de 0,11 e 0,15 ano para homens e mulheres, respectivamente. As mortes com causas mal-definidas, ao contrário do primeiro momento examinado, passaram a contribuir positivamente com a elevação da esperança de vida ao nascer dos homens nos anos de 2000 a 2005.
A Tabela 4 mostra que as idades de 45-79 anos foram as maiores beneficiadas pela redução dos níveis de mortalidade por doenças do aparelho circulatório nos anos 90 , respondendo por $68,8 \%$ do ganho total do tempo médio de vida da população masculina e por $71,3 \%$ da feminina. No caso das doenças respiratórias, as idades menores de 15 anos foram as principais responsáveis pelo aumento da esperança de vida, tanto da população masculina quanto da feminina.

No conjunto das causas externas, foi a redução da mortalidade nas idades de 15-44 anos que mais incrementou o tempo médio de vida da população masculina entre 2000 e 2005, sendo que a maior contribuição (1,54 ano) pertence ao grupo etário de 15-29 anos. Na esperança de vida ao nascer das mulheres, a maior contribuição destas causas ocorreu nas idades menores que 30 anos com 0,26 ano, o que equivale a $83,7 \%$ do total de aumento gerado pelas causas externas.

A contribuição negativa das neoplasias no aumento da esperança de vida dos homens entre 2000 e 2005 se distribuiu nas idades de 15-29 e nas maiores de 45 anos. Entre as mulheres, o 
Contribuição em número de anos e em porcentagem dos grupos de causas de morte no aumento da esperança de vida ao nascer nos períodos de 1991 a 2000 e 2000 a 2005, segundo o sexo. Campinas, São Paulo, Brasil, 1991,2000 e 2005.

\begin{tabular}{|c|c|c|}
\hline \multirow[t]{2}{*}{ Sexo/Causas de morte } & $1991-2000$ & $2000-2005$ \\
\hline & Anos (\%) & Anos (\%) \\
\hline \multicolumn{3}{|l|}{ Homens } \\
\hline Doenças infecto-parasitárias & $0,2537(12,3)$ & $0,2057(6,2)$ \\
\hline Neoplasias & $0,2838(13,7)$ & $-0,1120(-3,4)$ \\
\hline Doenças do aparelho circulatório & $1,3678(66,1)$ & $0,3631(10,9)$ \\
\hline Doenças do aparelho respiratório & $0,5294(25,6)$ & $0,3182(9,6)$ \\
\hline Causas mal-definidas & $-0,0167(-0,8)$ & $0,2242(6,7)$ \\
\hline Causas externas & $-1,0641(-51,4)$ & $2,3209(69,7)$ \\
\hline Demais causas & $0,7158(34,6)$ & $0,0109(0,3)$ \\
\hline Todas & $2,0695(100,0)$ & $3,3310(100,0)$ \\
\hline \multicolumn{3}{|l|}{ Mulheres } \\
\hline Doenças infecto-parasitárias & $0,0753(2,3)$ & $0,0419(4,7)$ \\
\hline Neoplasias & $0,3439(10,7)$ & $-0,1478(-16,6)$ \\
\hline Doenças do aparelho circulatório & $1,4053(43,5)$ & $0,3770(42,5)$ \\
\hline Doenças do aparelho respiratório & $0,3729(11,6)$ & $0,1698(19,1)$ \\
\hline Causas mal-definidas & $0,0447(1,4)$ & $0,1194(13,4)$ \\
\hline Causas externas & $0,0725(2,2)$ & $0,3114(35,1)$ \\
\hline Demais causas & $0,9137(28,3)$ & $0,0159(1,8)$ \\
\hline Todas & $3,2283(100,0)$ & $0,8877(100,0)$ \\
\hline \multicolumn{3}{|l|}{ Ambos os sexos } \\
\hline Doenças infecto-parasitárias & $0,1738(6,6)$ & $0,1306(5,9)$ \\
\hline Neoplasias & $0,3177(12,0)$ & $-0,1295(-5,8)$ \\
\hline Doenças do aparelho circulatório & $1,4023(52,9)$ & $0,3769(17,0)$ \\
\hline Doenças do aparelho respiratório & $0,4692(17,7)$ & $0,2526(11,4)$ \\
\hline Causas mal-definidas & $0,0116(0,4)$ & $0,1771(8,0)$ \\
\hline Causas externas & $-0,5418(-20,5)$ & $1,3945(62,9)$ \\
\hline Demais causas & $0,8161(30,8)$ & $0,0136(0,6)$ \\
\hline Todas & $2,6488(100,0)$ & $2,2159(100,0)$ \\
\hline
\end{tabular}

impacto negativo concentrou-se nas idades acima de 45 anos.

\section{Discussão}

A esperança de vida ao nascer da população residente no Município de Campinas em 2005 era de 74,7 anos. Este valor é superior ao registrado para o país em 2008, que foi de 72,7 anos 10. O Estado de São Paulo também somente alcançou um patamar próximo ao da população de Campinas no ano de 2008, quando a longevidade foi de 74,5 anos de vida.

A evolução da esperança de vida ao nascer da população de Campinas entre 1991 e 2005, que resultou num importante incremento de 4,8 anos, é reflexo da redução diferenciada dos níveis de mortalidade tanto em relação aos grupos etários, quanto às causas de morte. Com a aplicação do método de Pollard 7 , que mensura as variações da mortalidade sobre os ganhos na esperança de vida ao nascer, o presente artigo revelou o quanto cada faixa etária e grupo de causas de óbitos contribuíram para este aumento.

Em Campinas, graças à alta cobertura do registro de eventos vitais e à inexistência de razões para se supor que haja subnotificação diferencial, não foi utilizado nenhum tipo de técnica de correção dos dados para o cálculo dos coeficientes de mortalidade. Com o objetivo de minimizar os efeitos de oscilação aleatória dos dados, foi empregada a média trienal dos óbitos nas estimativas dos coeficientes de mortalidade por grupo etário e por causa de morte para a população maior de 1 ano de idade. Também 
Contribuição em número de anos dos grupos de causas de morte no aumento da esperança de vida ao nascer, nos períodos de 1991 a 2000 e 2000 a 2005 , segundo grupo etário e sexo. Campinas, São Paulo, Brasil, 1991, 2000 e 2005.

\begin{tabular}{|c|c|c|c|c|c|c|c|c|}
\hline $\begin{array}{l}\text { Sexo/Grupos } \\
\text { etários (anos) }\end{array}$ & $\begin{array}{c}\text { Doenças } \\
\text { infecto- } \\
\text { parasitárias }\end{array}$ & Neoplasias & $\begin{array}{c}\text { Doenças do } \\
\text { aparelho } \\
\text { circulatório }\end{array}$ & $\begin{array}{l}\text { Doenças do } \\
\text { aparelho } \\
\text { respiratório }\end{array}$ & $\begin{array}{l}\text { Causas mal- } \\
\text { definidas }\end{array}$ & $\begin{array}{l}\text { Causas } \\
\text { externas }\end{array}$ & $\begin{array}{l}\text { Demais } \\
\text { causas }\end{array}$ & Total \\
\hline \multicolumn{9}{|l|}{$1991-2000$} \\
\hline Homens & 0,2537 & 0,2838 & 1,3678 & 0,5294 & $-0,0167$ & $-1,0641$ & 0,7158 & 2,0695 \\
\hline $0-14$ & 0,1518 & 0,0484 & 0,0589 & 0,1663 & $-0,0194$ & 0,0657 & 0,3400 & 0,8118 \\
\hline $15-29$ & 0,1781 & $-0,0067$ & 0,0606 & 0,0790 & 0,0133 & $-0,8791$ & 0,0573 & $-0,4974$ \\
\hline $30-44$ & $-0,0230$ & 0,0137 & 0,1615 & 0,0618 & $-0,0018$ & $-0,3024$ & 0,0884 & $-0,0017$ \\
\hline $45-59$ & $-0,0032$ & 0,1719 & 0,3539 & 0,0855 & $-0,0209$ & $-0,0009$ & 0,1262 & 0,7124 \\
\hline $60-79$ & $-0,0349$ & 0,0973 & 0,5879 & 0,1153 & 0,0159 & 0,0379 & 0,0927 & 0,9121 \\
\hline 80 ou mais & $-0,0152$ & $-0,0409$ & 0,1450 & 0,0214 & $-0,0039$ & 0,0147 & 0,0112 & 0,1323 \\
\hline Mulheres & 0,0753 & 0,3439 & 1,4053 & 0,3729 & 0,0447 & 0,0725 & 0,9137 & 3,2283 \\
\hline $0-14$ & 0,1094 & $-0,0062$ & 0,0260 & 0,2074 & $-0,0109$ & 0,0580 & 0,5455 & 0,9292 \\
\hline $15-29$ & 0,0336 & $-0,0012$ & 0,0231 & 0,0409 & 0,0009 & $-0,0059$ & 0,0771 & 0,1684 \\
\hline $30-44$ & $-0,0422$ & 0,0172 & 0,0512 & 0,0566 & 0,0007 & $-0,0179$ & 0,0529 & 0,1186 \\
\hline $45-59$ & 0,0034 & 0,0984 & 0,2085 & 0,0108 & $-0,0021$ & 0,0066 & 0,1073 & 0,4328 \\
\hline $60-79$ & $-0,0060$ & 0,2367 & 0,7942 & 0,0590 & 0,0399 & 0,0223 & 0,1612 & 1,3072 \\
\hline 80 ou mais & $-0,0229$ & $-0,0010$ & 0,3023 & $-0,0018$ & 0,0161 & 0,0095 & $-0,0302$ & 0,2720 \\
\hline \multicolumn{9}{|l|}{$2000-2005$} \\
\hline Homens & 0,2057 & $-0,1120$ & 0,3631 & 0,3182 & 0,2242 & 2,3209 & 0,0109 & 3,3310 \\
\hline $0-14$ & 0,0174 & 0,0080 & 0,0039 & 0,0707 & 0,0496 & 0,0783 & 0,0699 & 0,2978 \\
\hline $15-29$ & 0,0367 & $-0,0013$ & 0,0201 & 0,0115 & 0,0192 & 1,5418 & $-0,0084$ & 1,6196 \\
\hline $30-44$ & 0,1178 & 0,0434 & 0,0721 & 0,0585 & 0,0262 & 0,5812 & 0,1122 & 1,0115 \\
\hline $45-59$ & 0,0140 & $-0,1130$ & 0,1511 & 0,0796 & 0,0346 & 0,1161 & $-0,0172$ & 0,2652 \\
\hline $60-79$ & 0,0197 & $-0,0372$ & 0,2232 & 0,1321 & 0,0694 & 0,0184 & $-0,0578$ & 0,3678 \\
\hline 80 ou mais & 0,0000 & $-0,0120$ & $-0,1072$ & $-0,0342$ & 0,0253 & $-0,0150$ & $-0,0878$ & $-0,2309$ \\
\hline Mulheres & 0,0419 & $-0,1478$ & 0,3770 & 0,1698 & 0,1194 & 0,3114 & 0,0159 & 0,8877 \\
\hline $0-14$ & 0,0346 & 0,0123 & $-0,0080$ & 0,0650 & 0,0170 & 0,1142 & 0,1230 & 0,3582 \\
\hline $15-29$ & 0,0241 & 0,0121 & 0,0076 & 0,0095 & 0,0244 & 0,1465 & $-0,0079$ & 0,2163 \\
\hline $30-44$ & 0,0356 & 0,0010 & 0,0662 & 0,0104 & 0,0203 & 0,0609 & 0,0149 & 0,2092 \\
\hline $45-59$ & $-0,0101$ & $-0,0539$ & 0,0789 & 0,0509 & 0,0112 & 0,0073 & 0,0224 & 0,1065 \\
\hline $60-79$ & $-0,0210$ & $-0,0936$ & 0,2521 & 0,0891 & 0,0031 & 0,0125 & 0,0258 & 0,2680 \\
\hline 80 ou mais & $-0,0212$ & $-0,0257$ & $-0,0198$ & $-0,0551$ & 0,0435 & $-0,0299$ & $-0,1623$ & $-0,2706$ \\
\hline
\end{tabular}

a comparabilidade das duas revisões da CID foi garantida a partir da compatibilização realizada com a correspondência dos capítulos das versões da CID e com a modificação do código de AIDS nos atestados de óbitos do período em que vigorava a CID-9. Este procedimento foi necessário, uma vez que a AIDS constitui uma importante causa de morte em Campinas.

Em relação ao número de anos acrescentados na esperança de vida ao nascer em um intervalo de tempo, é preciso esclarecer, a priori, a diferença entre o valor obtido através da tábua de mortalidade e o estimado pelo método de Pollard. Ao assumir que não há mudanças dos níveis de mortalidade em outras idades e, portanto, ao não considerar os efeitos de interação entre as reduções de mortalidade em diferentes idades, este método oferece uma estimativa aproximada 7 .

Os maiores ganhos de anos de vida, entre 1991 e 2000, concentraram-se nas idades extremas, sendo que o grupo de menores de 1 ano de idade foram os que mais contribuíram com o aumento da esperança de vida ao nascer em Campinas neste período. Resultado semelhante foi observado por Botega et al. 11 no Estado de Santa Catarina na década de 90, em cujo estudo também foi utilizado o método de Pollard. 
Entre 2000 e 2005, foram registrados padrões distintos da distribuição dos ganhos entre as idades conforme o sexo. Os ganhos da população masculina foram maiores nos jovens e adultos de 15-44 anos (79\%), ocasionando redução da contribuição dos menores de 1 ano de idade. Para as mulheres, os maiores incrementos são decorrentes do declínio da mortalidade entre os menores de 1 ano e nas idades de 25-39 e de 55-74 anos. À semelhança da situação de Campinas, Romero et al. 12 verificaram que, em Medellín na Colômbia entre os triênios 1989 a 1991 e 1994 a 1996, foram as idades intermediárias as maiores responsáveis pelo aumento da esperança de vida ao nascer masculina, ao passo que, entre as mulheres, as maiores contribuições se concentraram nas idades extremas.

Estudos sobre tendência dos ganhos de anos de vida em países desenvolvidos mostram que, com o aumento da esperança de vida ao nascer, as maiores contribuições se deslocam das idades mais jovens para os maiores de 65 anos de idade 1,5,13,14. Os dados de Campinas, ao indicarem a predominância do grupo de menores de 1 ano de idade entre as mulheres e a contribuição elevada nas idades intermediárias entre os homens, acenam para o quanto a esperança de vida ao nascer do município pode aumentar. O tempo médio de vida de Campinas no ano de 2005 já havia sido atingido pelo Japão em 1980, período no qual neste país as idades mais velhas passam a responder pelos maiores ganhos de anos de vida 13. Tal comparação serve para indicar a possível futura tendência do comportamento das idades em relação às contribuições para o aumento da expectativa de vida ao nascer de Campinas.

As doenças infecciosas e parasitárias apresentaram redução das probabilidades de morte, acarretando ganhos de anos de vida ao longo do período analisado. Todavia, o impacto negativo destas causas de mortes em alguns agrupamentos etários sobre a variação da vida média ao nascer pode ter sido gerado por algumas doenças evitáveis, como a septicemia e a AIDS. Em estudo sobre a evolução da mortalidade por causas de óbitos evitáveis e não evitáveis e o seu impacto nos ganhos da esperança de vida nas Regiões Metropolitanas de Belo Horizonte (Minas Gerais) e Salvador (Bahia) entre 1985 e 1995, no qual foi empregado o método de Pollard, Abreu \& Rodrigues 15 destacaram o impacto negativo da AIDS sobre a variação do tempo médio de vida de homens e mulheres, sobretudo, nos jovens e adultos. Estes dados reforçam achados do estudo de Buchalla et al. 16, os quais apontam que, apesar da acentuada redução dos coeficientes de mortalidade por diversas doenças infecciosas ao longo do século XX, ainda permanecem frequentes as mortes por septicemia, tuberculose e doença de Chagas e que, principalmente, a emergência da AIDS nos anos 80 tem sido responsável por elevado número de óbitos. Também na França 17 e na Itália 18,19, a AIDS é apontada como importante causa, entre as doenças infecciosas, na redução dos ganhos da esperança de vida ao nascer. Na Rússia, além da AIDS, verificou-se que a tuberculose ocasionou perdas de anos de vida entre 1965 e 199920.

A maior contribuição no aumento da expectativa de vida ao nascer entre 1991 e 2000 foi gerada pelas doenças do aparelho circulatório. O declínio de mortalidade por estas doenças resultou na elevação de 1,4 ano de sobrevida, correspondendo a mais da metade do ganho global deste período. Este incremento foi proporcionado enormemente pelo grupo de 45-79 anos. Tais resultados são consistentes aos observados na literatura. Em Santa Catarina nos anos 90, a principal contribuição positiva na evolução da esperança de vida ao nascer deveu-se às doenças circulatórias, sendo que os maiores ganhos estavam concentrados nas idades mais avançadas e eram crescentes com o aumento da idade 11 . No Japão, entre 1970 e 2000, as doenças cardiovasculares, em particular as cerebrovasculares, proporcionaram o maior ganho na esperança de vida ao nascer, e os grupos etários com mais de 65 anos foram os principais responsáveis por este aumento ${ }^{13}$. Na variação da expectativa de vida da Alemanha entre 1962 e 2002, as doenças cardiovasculares contribuíram substancialmente com 2,7 anos entre os homens e 3,0 anos entre as mulheres 1 .

A tendência de queda dos níveis de mortalidade por doenças do aparelho circulatório em Campinas, registrada ao longo destes 15 anos estudados, corresponde à situação brasileira para a qual as estatísticas apontavam a origem do declínio da mortalidade por estas causas em meados dos anos 80 21,22. Uma vez que os índices de prevalência das doenças cardiovasculares aumentam com a idade devido ao envelhecimento relativo da população e à maior longevidade 23 , a explicação para esta queda da mortalidade e o consequente aumento do número médio de anos de vida parece estar associada às melhores condições de assistência médica e ao avanço de recursos tecnológicos para prevenção e tratamento, postergando a idade do óbito e contribuindo com o decréscimo da letalidade destas causas $11,21,24$. Possivelmente, a maior procura por serviços de saúde, obtida graças às campanhas e programas de saúde específicos às doenças cardiovasculares, além de diversas intervenções de saúde que priorizam o combate aos principais fatores de risco para doenças crônicas (como o 
tabagismo, dieta inadequada e sedentarismo) e que incentivam mudanças de comportamentos 25,26 , podem também ter favorecido a queda da mortalidade e o aumento da sobrevida.

$\mathrm{O}$ aumento dos coeficientes de mortalidade por neoplasias, entre 2000 e 2005, refletiu-se na perda de 0,11 e 0,15 ano no incremento na esperança de vida ao nascer de homens e mulheres, respectivamente. Nos países desenvolvidos, ao contrário, as neoplasias, embora em menor proporção, contribuem positivamente com a ampliação dos anos a serem vividos desde os anos 80 1,13,14. Analisando os tipos específicos de cânceres, Klenk et al. 1 afirmam que o câncer de estômago foi o maior responsável, entre estas doenças, pelo aumento da esperança de vida ao nascer na Alemanha entre 1962 e 2002. Em relação ao câncer de pulmão, estudos verificaram comportamento diferente segundo o sexo na variação da esperança de vida, tendo exercido impacto positivo entre os homens e negativo entre as mulheres 1,14,17,18. Para o Brasil, as estatísticas de incidência e de mortalidade por neoplasias revelam a complexidade e as dificuldades envolvidas no desenvolvimento de estratégias para o controle destas doenças, uma vez que coexistem no país tumores associados ao alto status socioeconômico (como o de próstata e cólon e reto) e às situações de pobreza (por exemplo, o de colo de útero) 27.

As doenças do aparelho respiratório contribuíram com ganhos na esperança de vida ao nascer da população masculina e feminina, e apenas as idades de 80 anos ou mais não tiveram impacto positivo entre 2000 e 2005. Diferentemente de Campinas em que responderam por um expressivo aumento dos anos de sobrevida neste período $(9,6 \%$ entre os homens e $19,1 \%$ entre as mulheres), em Santa Catarina as doenças respiratórias foram responsáveis por apenas $4,1 \% \mathrm{e}$ $1,5 \%$ da variação da expectativa de vida ao nascer de homens e mulheres, respectivamente 11. Em estudo realizado na Holanda foi verificado que as doenças pulmonares obstrutivas crônicas produziram um declínio da ampliação de anos de vida na expectativa de vida aos 60 anos e aos 85 anos de idade 14 .

Diversas investigações nas áreas da epidemiologia e da demografia indicaram o vertiginoso crescimento da violência durante a década de 90 e os primeiros anos de 2000, registrando o rápido aumento da mortalidade 2,28 . Frente a esta expressiva importância, as causas violentas se tornaram alvo de interesse de pesquisadores e formuladores de políticas públicas que se utilizaram de diversos métodos e indicadores para registrar a tendência e o perfil das causas de morte e para identificar os grupos sociodemográficos com maior exposição. Mais especificamente, na investigação da relação entre a mortalidade por causas externas e a esperança de vida, vários estudos nacionais recorreram às correlações entre estes indicadores, às técnicas de Risco Competitivo em Tábuas de Vida de Múltiplo Decremento e de Anos Potenciais de Vida Perdidos 2,24,28,29. Pesquisas que empregaram o método de decomposição de Pollard também apontaram o forte impacto negativo das causas externas sobre a esperança de vida $3,11,12,15$.

A magnitude da influência das causas externas na esperança de vida ao nascer é traduzida, neste estudo, pela considerável perda de 1,06 ano na população masculina entre 1991 e 2000, sendo que nos grupos etários de 15-44 anos de idade as reduções foram mais intensas, o que revela a mortalidade precoce de jovens e adultos. Embora, entre as mulheres de 15-44 anos, as causas externas tenham reduzido o aumento da esperança de vida ao nascer, o impacto foi significativamente menor.

Estes resultados são semelhantes aos encontrados na literatura, na qual os homens, sobretudo jovens e adultos, são apontados como as principais vítimas fatais de violências e acidentes de trânsito 2,3,12,15,17,28,30. De fato, em Campinas, entre 1991 e 2000, a forte influência negativa das probabilidades de morte por causas externas de homens de 15-44 anos, além de ocasionar a perda de um ano de vida, ainda se expressa no discreto aumento da esperança de vida ao nascer: ao longo de quase uma década, foram apenas 1,8 ano acrescidos no tempo médio de vida dos homens. No período seguinte, esta tendência se inverte. A apreciável queda das probabilidades de morte por causas externas na população masculina contribuiu para um significativo aumento de 2,3 anos na longevidade. Ou seja, a redução da mortalidade por estas causas respondeu por cerca de $70 \%$ do ganho total da esperança de vida ao nascer masculina obtido entre 2000 e 2005 . As principais responsáveis pela ampliação dos anos de vida foram as idades de 15-44, que contribuíram com 2,1 anos.

$\mathrm{Na}$ população feminina, incrementos relativamente pequenos na esperança de vida ao nascer entre 2000 e 2005 indicam a reversão da tendência da diminuição dos níveis de mortalidade. Comportamento semelhante havia sido registrado por Ferreira \& Castiñeiras 29 já para o ano de 1996 para as mulheres paulistas. Os autores, analisando o crescimento do tempo médio de vida entre 1940 e 1996 no estado, explicavam que esta quebra da tendência da mortalidade poderia ser atribuída à evolução de algumas causas de morte, como os acidentes de trânsito e as neoplasias. Outros estudos também registraram 
uma desaceleração da esperança de vida feminina associada ao aumento da mortalidade por neoplasias, em especial de pulmão ${ }^{17}$, por doenças cardiovasculares e acidentes de trânsito ${ }^{18}$. A ligeira estagnação do crescimento da esperança de vida ao nascer feminina de Campinas entre 2000 e 2005 parece estar associada tanto à maior longevidade das mulheres que impõe maiores dificuldades ao aumento do número médio de anos de vida, quanto à crescente proporção de mulheres acima dos 60 anos que passam a ser mais expostas às doenças crônicas não transmissíveis cujo ritmo de queda da mortalidade apresenta-se mais lento por ser relativamente de difícil controle e eliminação. Para reverter este quadro de ganhos pouco expressivos, é de extre- ma importância orientar esforços no combate às causas de morte, cujas contribuições na variação da esperança de vida ao nascer sofreram redução no decorrer dos dois períodos investigados, como é o caso, sobretudo, das neoplasias e doenças cardiovasculares.

O presente estudo, ao apontar os agrupamentos etários e o conjunto de causas de morte que menos contribuíram ou mesmo que provocaram a perda de anos a serem acrescentados na esperança de vida ao nascer, podem auxiliar na orientação de programas e políticas públicas de saúde no combate a doenças e agravos que mais atingem cada sexo e faixa de idade, visando a redução dos riscos de mortalidade e contribuindo para o aumento da esperança de vida ao nascer.

\section{Resumo}

O objetivo do estudo foi examinar o impacto das mudanças na mortalidade por idades e causas de morte sobre o aumento da esperança de vida ao nascer no Município de Campinas, São Paulo, Brasil, entre 1991, 2000 e 2005. Foram construídas tábuas de vida. O método de Pollard foi aplicado para estimar as contribuições das idades e causas de morte na variação da longevidade. O grupo etário de 0-1 ano foi o que mais contribuiu com o aumento da vida média masculina (31,1\%) e feminina (22,9\%) em 1991/2000. Entre 2000 e 2005, as idades de 15-44 anos responderam por 79\% do ganho masculino. A maior contribuição entre 1991 e 2000 foi gerada pelas doenças cardiovasculares (66,1\% entre os homens e 43,5\% entre as mulheres). As causas externas subtraíram 1,1 ano entre os homens. Entre 2000 e 2005, com a queda da mortalidade por estas causas, a esperança de vida masculina aumentou em 2,3 anos. As neoplasias provocaram redução de 0,11 ano para homens e 0,15 ano para mulheres. Estes resultados podem auxiliar na orientação de políticas públicas de saúde para redução da mortalidade e aumento da esperança de vida ao nascer.

Esperança de Vida ao Nascer; Mortalidade; Causas de Morte; Grupos Etários

\section{Colaboradores}

A. P. Belon e M. B. A. Barros realizaram a proposta do artigo, revisão de literatura, análise dos dados e redação do texto.

\section{Agradecimentos}

À Coordenação de Aperfeiçoamento de Pessoal de Nível Superior (CAPES), pela bolsa de doutorado recebida por A. P. Belon. Ao Conselho Nacional de Desenvolvimento Científico e Tecnológico (CNPq), pela bolsa de produtividade de M. B. A. Barros. 


\section{Referências}

1. Klenk J, Rapp K, Büchele G, Keil U, Weiland SK. Increasing life expectancy in Germany: quantitative contributionsfrom changesinage-and disease-specific mortality. Eur J Public Health 2007; 17:587-92.

2. Simões CCS. Perfis de saúde e de mortalidade no Brasil: uma análise de seus condicionantes em grupos populacionais específicos. Brasília: Organização Pan-Americana da Saúde; 2002.

3. Yazaki LM. Causas de morte e esperança de vida ao nascer no Estado de São Paulo e Regiões, 19751983. São Paulo: Fundação Sistema Estadual de Análise de Dados; 1990.

4. Bourguignon F, Morrison C. Inequality among World citizens: 1820-1992. Am Econ Rev 2002: 92:727-44.

5. Oeppen J, Vaupel JW. Broken limits of life expectancy. Science 2002; 296:1029-31.

6. Riley JC. Introduction: a global revolution in life expectancy. In: Riley JC, editor. Rising life expectancy: a global history. Cambridge: Cambridge University Press; 2001. p. 1-31.

7. Pollard JH. The expectation of life and its relationship to mortality. J Inst Actuar 1982; 109:225-40.

8. Jannuzzi PM. Projeções populacionais para pequenas áreas: métodos e aplicações. Rio de Janeiro: Escola Nacional de Ciências Estatísticas, Instituto Brasileiro de Geografia e Estatística; 2006. (Textos para Discussão, 22).

9. Arriaga EE. El análisis de la población con microcomputadoras. Córdoba: Universidad Nacional de Córdoba; 2001.

10. Instituto Brasileiro de Geografia e Estatística. Aspectos demográficos. In: Instituto Brasileiro de Geografia e Estatística, organizador. Síntese de indicadores sociais: uma análise das condições de vida da população brasileira 2009. Rio de Janeiro: Instituto Brasileiro de Geografia e Estatística; 2009. p. 19-36. (Estudos \& Pesquisas: Informação Demográfica e Socioeconômica, 26).

11. Botega LA, Ribeiro MM, Machado CJ. O impacto de variações na mortalidade por idade e causas sobre os ganhos na esperança de vida ao nascer em Santa Catarina, Brasil, nos anos 90. Cad Saúde Pública 2006; 22:1079-88.

12. Romero HG, Bedoya GSE, Velásquez MEA, Marín MP. Cambio en la esperanza de vida según tres grandes grupos de causas de muerte en Medellín, Colombia, de 1989-1991 a 1994-1996. Rev Panam Salud Pública 2002; 12:305-12.

13. Yoshinaga $\mathrm{K}$, Une $\mathrm{H}$. Contributions of mortality changes by age group and selected causes of death to the increase in Japanese life expectancy at birth from 1950 to 2000. Eur J Epidemiol 2005; 20:49-57.

14. Nusselder WJ, Mackenbach JP. Lack of improvement of life expectancy at advanced ages in the Netherlands. Int J Epidemiol 2000; 29:140-8.

15. Abreu DMX, Rodrigues RN. Diferenciais de mortalidade entre as regiões metropolitanas de Belo Horizonte e Salvador, 1985-1995. Rev Saúde Pública 2000; 34:514-21.
16. Buchalla CM, Waldman EA, Laurenti R. A mortalidade por doenças infecciosas no início e no final do século XX no Município de São Paulo. Rev Bras Epidemiol 2003; 6:335-44.

17. Meslé F. Écart d'espérance de vie entre les sexes: les raisons du recul de l'avantage féminin. Rev Epidemiol Sante Publique 2004; 52:333-52.

18. Conti S, Farchi G, Masocco M, Minelli G, Toccaceli V, Vichi M. Gender differentials in life expectancy in Italy. Eur J Public Health 2003; 18:107-12.

19. Conti S, Farchi G, Masocco M, Toccaceli V, Vichi M. The impact of the major causes of death on life expectancy in Italy. Int J Epidemiol 1999; 28:905-10.

20. Andreev EM, Nolte E, Shkolnikov VM, Varavikova E, McKee M. The evolving pattern of avoidable mortality in Russia. Int J Epidemiol 2003; 32:437-46.

21. Lima-Costa MF, Peixoto SV, Giatti L. Tendências da mortalidade entre idosos brasileiros (1980-2000). Epidemiol Serv Saúde 2004; 13:217-28.

22. Mansur AP, Favarato D, Souza MFM, Avakian SD, Aldrighi JM, Cesar LAM, et al. Tendência do risco de morte por doenças circulatórias no Brasil de 1979 a 1996. Arq Bras Cardiol 2001; 76:504-10.

23. Barros MBA, César CLG, Carandina L, Torre GD. Desigualdades sociais na prevalência de doenças crônicas no Brasil, PNAD-2003. Ciênc Saúde Coletiva 2006; 11:911-26.

24. Barboni AR, Gotlieb SLD. Impacto de causas básicas de morte na esperança de vida em Salvador e São Paulo, 1996. Rev Saúde Pública 2004; 38:16-23.

25. Malta DC, Castro AM, Gosch CS, Cruz DKA, Bressan A, Nogueira JD, et al. A Política Nacional de Promoção da Saúde e a agenda da atividade física no contexto do SUS. Epidemiol Serv Saúde 2009; 18:79-86.

26. Monteiro CA, Cavalcante TM, Moura EC, Claro RM, Szwarcwald CL. Population-based evidence of a strong decline in the prevalence of smokers in Brazil (1989-2003). Bull World Health Organ 2007; 85:527-34.

27. Guerra MR, Moura Gallo CV, Mendonça GAS. Risco de câncer no Brasil: tendências e estudos epidemiológicos mais recentes. Rev Bras Cancerol 2005; 51:227-34

28. Aidar T. O impacto das causas violentas no perfil de mortalidade da população residente no Município de Campinas: 1980 a 2000. Rev Bras Estud Popul 2003; 20:281-302.

29. Ferreira CEC, Castiñeiras LL. Está diminuindo a esperança de vida paulista? In: Anais do XI Encontro Nacional de Estudos Populacionais da ABEP. Belo Horizonte: Associação Brasileira de Estudos Populacionais; 1998. p. 1961-74.

30. Laurenti R, Jorge MHPM, Gotlieb SLD. Perfil epidemiológico da morbi-mortalidade masculina. Ciênc Saúde Coletiva 2005; 10:35-46.

Recebido em 02/Jun/2010

Versão final reapresentada em 10/Fev/2011 Aprovado em 24/Fev/2011 